Pinto, C. J. (2017). Valoración de credit default swap aplicación del modelo de Jarrow y Turnbull en un bono de deuda privada en Colombia. Revista Lebret 9. Bucaramanga, Colombia: Universidad Santo Tomás. pp 151-170 - ISSN Versión Impresa: 2145-5996 - ISSN Versión Web On-line: 2357-5468 - http://dx.doi.org/10.15332/rl.v0i9.1954

\title{
Valoración de credit default swap aplicación del modelo de Jarrow y Turnbull en un bono de deuda privada en Colombia*
}

\section{Valuation of credit default swap application of the Jarrow-Turnbull model on a private debt bond in Colombia}

Carlos Javier Pinto Suárez

\begin{abstract}
Resumen
El estudio presenta la aplicación empírica de un modelo de forma reducida para la estimación de probabilidades default, el cual ofrece bondades frente a otros modelos, porque permite no solo utilizarse para el cálculo de la probabilidad de default, sino también para la valoración del derivado de crédito Credit Default Swap - CDS desde el punto de vista del comprador, encontrándose oportuno el uso de este modelo cuando el mercado se caracteriza por su baja liquidez e insuficiente información estadística. Como base de aplicación del modelo se utilizó la información de un bono de referencia con vencimiento en cinco años de la empresa Ecopetrol, con el que se muestra el procedimiento para la estimación de la prima del CDS, lo que permitió hacer comparaciones entre modelo teórico y los valores disponibles en el mercado, encontrándose como resultado un valor muy similar entre estos y demostrar que el modelo se ajusta para estimar la probabilidad anual base, la probabilidad default acumulada de CDS, la prima de CDS en bonos privados, información que serviría de referencia a inversionistas para gestionar el riesgo default en bonos privados en Colombia a través del uso CDS.
\end{abstract}

\section{Palabras clave}

Riesgo default, valoración de Credit Default Swaps.

\section{Códigos de clasificación JEL: G11, G12, G24}

\begin{abstract}
The study presents the empirical application in reduced form of a model for the estimation of default probabilities, which offers benefits compared to other models because it can be used not only for the calculation of the probability of non-compliance but also for the valuation of the derivative of CDS credit from the buyer's point of view. Using this model is appropriate when the market is characterized by low liquidity and insufficient statistical information. As the basis for the model's application, the information on a five-year benchmark bond of the Ecopetrol oil company has been used, to show the procedure for the estimation of the CDS premium. This allowed making comparisons between the theoretical model and values available on the market. The result has been a very similar value between them, and it is shown that the model fits to estimate the annual base probability, the cumulative CDS default probability, and the CDS premium on private bonds. This information could serve as a reference for investors to manage default risk on private bonds in Colombia using CDS.
\end{abstract}

\section{Keywords}

Default risk, valuation of Credit Default Swaps.

* Artículo derivado del proyecto de maestría en Finanzas, titulado "Estrategia de cobertura de riesgo default a partir de la aplicación del derivado Credit Default Swap para emisión de deuda privada en Colombia”.

1 Magíster en Finanzas. Docente Facultad de Negocios Internacionales Universidad Santo Tomás Bucaramanga. Correo electrónico: carlos.pinto@ustabuca.edu.co 


\section{Introducción}

El desarrollo del mercado de derivados de crédito si bien es un fenómeno de los últimos años, la transferencia de riesgo de crédito no es un fenómeno reciente. Como lo menciona Fradique-Méndez (2008) desde la década de los setenta, con el aumento progresivo de las necesidades de las transacciones entre contrapartes de diversas jurisdicciones y ante las necesidades de cobertura por el desarrollo del mercado de divisas y las volatilidades existentes en las tasas de cambio, esto trajo consigo el uso común de una serie de contratos, los cuales se caracterizaron por tener cláusulas en los que procuraban incorporar las situaciones de default requeridas, estos contratos con el tiempo fueron más sofisticados y pasaron de un uso mayoritariamente para cobertura a uno especulativo, aunado a la falta de mecanismos de control en el mercado OTC, desencadenó en la última crisis financiera de 2008 y puso de manifiesto la importancia del control y de la correcta medición del riesgo de crédito en especial en el ámbito bancario, por los efectos sistémicos que pueden generarse dentro de un proceso de gestión inapropiado de los instrumentos financieros, lo que dejó al descubierto las graves deficiencias que en materia de gestión de riesgos existían en el sistema bancario específicamente.

Por tanto, se hace necesaria la adopción de medidas que permitan minimizar el impacto del riesgo de crédito que afecta las inversiones. Entre los instrumentos que se encuentran en el mercado existen los derivados de crédito, que están obteniendo cada vez mayor importancia por su uso, en razón a la incertidumbre ocasionada por el cambiante entorno internacional, que, junto a factores internos del país, generan pérdida de confianza a los inversionistas respecto al cumplimiento de sus políticas de riesgo crediticio, en especial, en inversiones de deuda corporativa, en el que el nivel de riesgo es mayor frente a la deuda pública.

En este sentido es necesario precisar que la asignación de riesgo de crédito a emisiones de deuda se ve reflejado cuantitativamente en un mayor valor de la tasa de interés por ofrecer en el valor emitido. Según Labanda (2005) como el inversionista no es neutral al riesgo, exige un diferencial por encima de la tasa libre de riesgo. El valor del spread de crédito (diferencial sobre la tasa libre de riesgo) estaría ligado a la calidad del crédito (definido por la probabilidad de incumplimiento) y por la estimación de pérdida en caso de default. El spread de crédito del mercado representa entonces una estimación de la pérdida por riesgo de crédito esperada, que a su vez incorpora aspectos no ligados al crédito, como liquidez y aspectos regulatorios, convirtiéndose en una variable determinante en el estudio del riesgo de crédito.

Dentro de los instrumentos financieros que aprueben gestionar el riesgo de crédito se encuentran una variedad de derivados de crédito, que cuentan con una serie de características que permiten separar, valorar, replicar, transmitir y asegurar la totalidad o una parte del riesgo de crédito de un activo financiero, mediante contratos cuya expresión hace referencia a distintas clases de opciones y permutas, cuyo 
riesgo de crédito procede de un activo financiero, a cambio de una prima, o de un flujo monetario y durante un período de tiempo igual o inferior a la vida del activo subyacente (Pérez, 2002).

Así mismo estos autores refieren una clasificación en tres grupos de instrumentos en los que está basada la mayor parte de las diferentes fórmulas contractuales: las permutas crediticias (Credit Swap), las opciones crediticias (Credit Options) y los productos estructurados que incluyen derivados crediticios.

Dentro de la categoría de Credit Swap existen muchos instrumentos, los cuales se identifican porque comparten características básicas. Algunos de estos instrumentos son los Total Return Swaps (TRS) y los Asset Swaps, que a su vez presentan tres variedades (Par Asset Swap, Cross-Currency Asset Swap, Market Asset Swap) y los Credit Default Swaps, en adelante CDS, que son el objeto de estudio por ser los más utilizados en la actualidad como lo indican los montos de negociación seguidamente.

El desarrollo del mercado de CDS se ha dado en el mercado extrabursatil (over the counter) en adelante OTC, este presenta un marcado crecimiento desde el año 2000 en comparación con los demás derivados de crédito, al alcanzar "más del 70\% con vencimientos entre 3 y 5 años sobre nombres de referencia de calidad "Investment Grade"; sin embargo, se vio afectado de manera negativa desde la crisis financiera de 2008, como se evidencia en la figura 1, cuando el volumen de nocionales del mercado mundial disminuyó, tanto en el volumen de los CDS de nocionales bruto sencillo o single-name al pasar de 15 billones de dólares aproximadamente en octubre de 2008 a 8 billones de dólares aproximadamente en enero de 2015, como en el total de CDS al pasar de 33,5 billones de dólares aproximadamente hasta 15,4 billones, respectivamente.

Figura 1. Evolución de los montos nocionales de los CDS 2008 - 2014

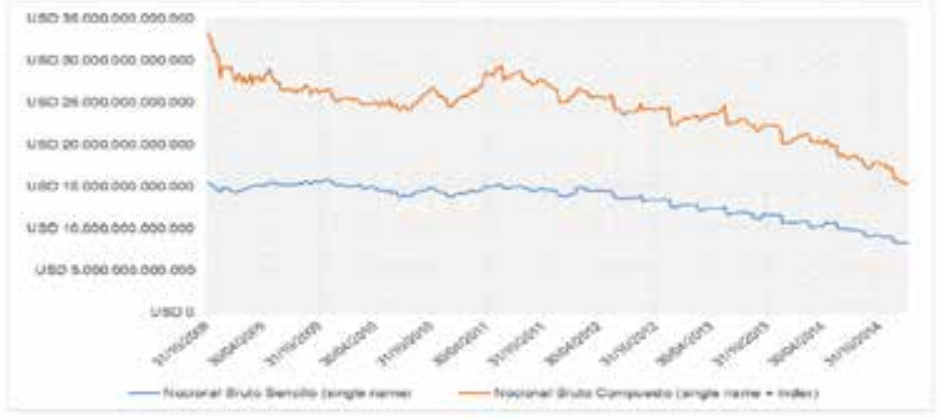

Fuente: Elaboración propia datos tomados de ISDA (2015). 
Una parte del auge del mercado de CDS puede ser atribuido a la utilidad en la negociación generada por las entidades financieras, las cuales, en lugar de utilizarlos con fines de cobertura, los emplean con fines especulativos o de arbitraje, al ocupar una posición predominante tanto como oferente como demandante de CDS, al tener a los intermediarios (dealers) más activos en estos mercados, y presentar un elevado grado de concentración $95 \%$ en 2009 de los valores nominales de todos los contratos establecidos y no liquidados de CDS tanto comprados como vendidos, frente a un número relativamente reducido de grandes corporaciones, referidas como estadísticas globales por Arce, González y Sanjuán (2010).

En cuanto al mercado de derivados de crédito en Colombia, es relevante precisar que estos instrumentos no son usados de manera habitual en el mercado de renta fija nacional, entre otras, por la baja liquidez que presenta el mercado y la restricción debido a la prohibición legal para la negociación de estos instrumentos en el país como lo expresa la Circular Reglamentaria Externa DODM 144 de 2006 del Banco de la República de Colombia; aunque posteriormente otra norma permitió a las entidades financieras realizar ciertas operaciones con derivados de crédito, cuando cumplan con los criterios establecidos en el capítulo XVIII de la Circular Externa 013 de junio de 2006 de la Superintendencia Financiera de Colombia.

Por todo lo anterior, resulta significativo estudiar las aplicaciones en el mercado financiero colombiano de herramientas de cobertura para emisiones de deuda como los Credit Default Swap (CDS), lo que podría facilitar el desarrollo de la negociación de estos instrumentos y los beneficios en la gestión de riesgo default, en especial, para inversionistas en deuda privada, al poder mantener o mejorar la calificación del emisor.

Los CDS están considerados dentro de los derivados de crédito y en esencia, es un contrato que se utiliza como instrumento para cubrirse ante el posible incumplimiento de su contraparte con las obligaciones contractuales crediticias durante la vigencia del contrato; por lo que se pretende es transferir este riesgo a un tercero a modo de protección, sin que necesariamente deba transferirse o negociarse el activo de referencia, tal como se representa en la operación crediticia de la figura 2 , en la cual el activo de referencia no se traslada de la operación crediticia hacia la operación de cobertura en la que se negocia el CDS.

En la tipología más común de los contratos CDS, el comprador pretende cubrirse al adquirir una opción de incumplimiento, que obligue a un tercero (vendedor de protección), a pagarle el dinero que compense la pérdida del valor nominal del activo de referencia durante el plazo convenido o antes, en caso que ocurra el incumplimiento o default. Este incumplimiento se reconoce siempre y cuando esté clasificado dentro de los eventos de crédito pactados con la entidad de referencia. En caso de que no ocurra evento de crédito, el vendedor de la protección no tiene obligación de pago, y por su parte, el comprador de protección se obliga a pagar una prima en dinero de manera periódica durante la vigencia de la protección de acuerdo con el contrato. 
Figura 2. Esquema funcional del CDS

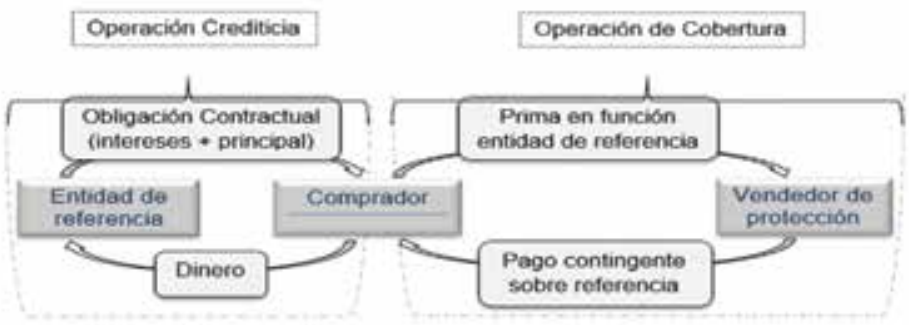

Fuente: Elaboración propia.

Por tanto, se hace necesario para los operadores de este mercado encontrar medidas eficientes para cuantificar tanto los riesgos como las pérdidas y la probabilidad de que esto ocurra, para ello se intentan una serie de aproximaciones para poder juzgar el nivel de riesgo default. El default puede tener causas de diversa naturaleza y esta diversidad de factores hace que la predicción del momento en que se produzca el default sea compleja.

La evidencia disponible sugiere que la valoración del riesgo default determina en gran medida las primas crediticias, por tanto, un factor fundamental para el desarrollo de un mercado de CDS consiste en lograr el perfeccionamiento en la valoración de este riesgo, para que los precios de los instrumentos de cobertura reflejen adecuadamente dicho riesgo. Por todo lo anterior se plantea el establecer cuál de las diversas teorías de estimación de probabilidad default será la apropiada para valorar este riesgo, respecto de las empleadas en la negociación en los mercados de derivados de crédito, lo que permitirá determinar si el valor a pagar por la prima establecida en el mercado de derivados corresponde a una realidad económica del mercado para el tomador del instrumento.

La literatura en esta área ha desarrollado modelos que se agrupan principalmente en tres enfoques teóricos para el riesgo de default, conocidos como modelos estructurales, modelos de forma reducida y modelos de información incompleta.

Según Arbeláez y Maya (2007), los modelos estructurales se basan en la evolución de las variables orgánicas de la empresa, como es el caso del valor de los activos y de la deuda. Los modelos denominados de información incompleta pretenden combinar la intuición económica que explica el riesgo de crédito, característica de los modelos estructurales, con la aplicabilidad empírica de los modelos de forma reducida. Estos modelos se caracterizan por no hacer supuestos acerca de la dinámica de la intensidad de default.

En cuanto a los modelos de forma reducida, por el contrario, no se enfocan en factores tales como la estructura de capital de la empresa, sino en el riesgo de default 
inherente a los precios de los activos financieros que son emitidos por ella, ya que no toman en cuenta de forma explícita la relación entre el incumplimiento y las variables económicas de la empresa como la estructura de capital, sino que introducen de forma separada la probabilidad de incumplimiento y la tasa de recuperación. La modelación de este evento dependerá directamente de la probabilidad de default de los instrumentos financieros de la empresa, a través de un proceso estocástico, lo que permite calibrar su probabilidad de ocurrencia directamente, a partir de los precios del mercado.

En estos modelos las probabilidades de default son descritas como un proceso exógeno, que en la mayoría de los casos se calcula desde de la prima de riesgo crediticio, lo que a su vez se determina a partir de los precios de los bonos negociados en los mercados financieros. También se utilizan otras variables exógenas entre las que podemos mencionar los precios de los bonos de las empresas, los diferenciales de los CDS o las calificaciones de crédito.

Estos modelos fueron introducidos en los años noventa por Litterman e Iben y Jarrow y Turnbull quienes estudian el caso donde existe un proceso Poisson con tasa de default constante y el pago conocido ante la ocurrencia del evento. Su trabajo especifica procesos exógenos para la evolución de la estructura temporal de la tasa libre de riesgo y la tasa del bono corporativo, para luego aplicar una metodología de valoración neutral al riesgo. Este análisis derivó en el modelo que Jarrow elaboró entre los años 1999 a 2001, cuya principal diferencia es que las probabilidades de default son una variable aleatoria que depende de otra variable aleatoria (los tipos de interés) y de un factor de riesgo que se distribuye de forma lognormal.

Así mismo, Duffie y Singleton (1999) presentan un modelo similar de forma reducida de un activo sujeto a riesgo de crédito para bonos corporativos y soberanos, en el cual argumentan que el spread existente entre un bono corporativo y un bono libre de riesgo se debe exclusivamente al riesgo de crédito. Aunque este puede deberse igualmente a otros factores tales como el riesgo de liquidez o asuntos impositivos, solamente se considera el riesgo de crédito para explicar la diferencia entre las rentabilidades de los bonos en este modelo.

Como una mejora Jarrow, Lando y Turnbull (1997) asumen un proceso de default que sigue una cadena de Markov y estiman los parámetros del proceso a partir de datos observados. El modelo emplea información sobre el historial de default y cambios en las calificaciones de riesgo.

En cuanto a la aplicación de los modelos en el mercado latinoamericano son pocos los estudios relacionados con Credit Default Swap, sin embargo, se encontró que en el trabajo de Arbeláez y Maya (2007) aplican el modelo de valoración reducida para (CDS) sobre bonos corporativos con base en el método de Simulación de Montecarlo y encuentran que el modelo propuesto tiene un mejor desempeño para la valoración de primas de CDS sobre títulos calificados con grado de inversión que los de grado 
de especulación. Además, se demuestra que el valor de las primas de los CDS no es muy sensible a cambios en la tasa de recuperación y en la tasa de interés libre de riesgo. Y el estudio de Sasaki, Chela y Kimura (2012), quienes presentan algunas de las características y modelos para el análisis del CDS y su aplicación en el mercado de Brasil. En cuanto a estudios relacionados con la aplicación de los modelos de Jarrow y Turnbull no se encontró información alguna.

En síntesis, se encontró que los modelos de forma reducida ofrecen ventajas adicionales como las mencionadas por Conde, Malacrida y Selves (2003), estos modelos requieren relativamente menos información que los estructurales, por lo que pueden utilizarse para valuar instrumentos con riesgo de crédito. Por su parte, Arbeláez y Maya (2007) resaltan la facilidad de calibración e implementación, en comparación con el poco éxito de las predicciones basadas en modelos estructurales. Así mismo, una diferencia sustancial se encuentra en que la mayoría de los modelos son aplicables para la estimación del default, pero no para la valoración de derivados financieros como el CDS, pues solo se encontraron tres modelos, los cuales en su mayoría se basan en modelos de forma reducida, que permiten las dos estimaciones, entre los que se encuentra el de Jarrow y Turnbull (1995).

\section{El modelo de valoración de Credit Default Swap}

El presente estudio plantea determinar la valoración de un CDS para un bono corporativo a partir de la aplicación empírica del modelo de Jarrow y Turnbull (1995), se parte de la obtención de los datos de entrada requeridos por el modelo disponible en el mercado colombiano, posteriormente se obtiene el spread o prima al resolver la ecuación con sus dos posibles ramas o patas, una representa los flujos por pagos de las primas del que se quiere cubrir (comprador) y la otra a los flujos esperados para recuperar por parte del emisor del CDS (vendedor) en caso de default y cada una de las ramas con una probabilidad de ocurrencia, los cuales no son simultáneos hasta su vencimiento a cada una de las ramas, para ello se emplea un modelo financiero en excel que estima el valor presente de los flujos futuros esperados, multiplicando cada flujo futuro por la probabilidad neutral al riesgo de su ocurrencia, el modelo relaciona la tasa de recuperación y los spreads cotizados en mercado bajo supuestos de riesgo neutral que corresponde a la diferencia de tasas entre bono con riesgo y bono sin riesgo, como se evidencia en Bonás, Llanes, Usón y Veiga (2007). Al final se calcula para un valor nocional y un tiempo obtenido del bono corporativo de referencia por cubrir, como resultado de la aplicación del modelo se podrá obtener una igualdad o una diferencia negativa o positiva, si hay diferencia se establece que el precio no está en equilibrio y se contrastará con el valor de mercado del spread de negociación del CDS para determinar si estos precios son justos o se ajustan a una valoración teórica. 
Para aplicar el modelo es necesario profundizar en las siguientes nociones; los CDS presentan dos posibles escenarios, cada uno con una cierta probabilidad de ocurrencia, los cuales no son simultáneos hasta su vencimiento:

- La no ocurrencia de default por parte de la referencia, por tanto, el comprador pagará la prima o spread acordado con el vendedor.

- La ocurrencia de un default por parte de la referencia, según el tipo de liquidación pactado. Si es física, el comprador entrega el bono de referencia y recibe el valor nominal de mismo por parte del vendedor; si es liquidación por diferencia, el comprador recibirá la diferencia entre el valor del bono y su valor de mercado después de default. El contrato debe especificar el pago de una prima o spread por parte del comprador de protección.

La figura 3 presenta los dos escenarios habituales, donde $\mathrm{p}$ es la probabilidad de default a vencimiento, $\mathrm{S}$ es el valor de la prima y $\mathrm{R}$ es la tasa estimada de recuperación de la referencia.

Figura 3. Esquema simple de posibles escenarios para el comprador de un CDS

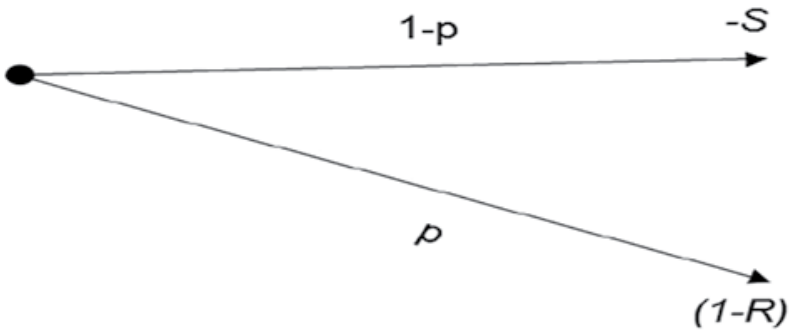

Fuente: Elaboración propia.

A partir del anterior esquema se puede comprender mejor la aplicación de la valoración del modelo desde la perspectiva del comprador de la protección, expresado como el valor esperado de los flujos futuros descontados.

Para realizar la valoración, el modelo de Jarrow y Turnbull se rige por varios supuestos entre los que se encuentran la tasa de recuperación constante $(R)$; el valor por reclamar, que es el nominal más los intereses acumulados hasta el suceso de crédito; el spread $(s)$, determinado por valoración neutral al riesgo, los inversores neutrales al riesgo, la ausencia de oportunidades de arbitraje; las diferentes tasas son independientes a cualquier variable de estado y al momento del suceso de crédito.

El supuesto la probabilidad neutral al riesgo se determinada entonces por la diferencia entre un bono con riesgo de incumplimiento y un bono libre de riesgo con 
igual periodicidad, donde se considera al spread como la diferencia de las rentabilidades entre ambos bonos $e^{-r}$, la cual se debe únicamente al riesgo de crédito.

En definitiva, para proceder a valorar un CDS desde la perspectiva del comprador, se inicia de la versión simple del modelo de un solo período y se estima la esperanza descontada de los pagos generados o payoff, con las siguientes expresiones:

Para el caso en el que no haya pagado la prima, ocurrido el default:

$$
C D S=-(1-p) * s * e^{-r}+(1-R) * p * e^{-r}
$$

Para el caso en el que se haya pagado la prima, ocurrido el default:

$$
C D S=-(1-p) * s * e^{-r}+(1-R-S) * p * e^{-r}
$$

Lo que sería la representación matemática para la valoración de un CDS en un período, como se presentó en la figura 1 con solo dos posibles escenarios.

En la práctica, en un contrato de CDS se determinan una serie de fechas en las que el comprador de protección se compromete a pagar la fracción de la prima convenida, siempre y cuando la entidad de referencia no haya presentado previamente default. Si se llegara a confirmar un evento de crédito, el comprador de protección deberá recibir del vendedor la compensación por el valor que no se recupera del bono de referencia.

Como respuesta a esta situación, se plantea una extensión al modelo simple anterior en la que exista más de un período de pago de primas, lo que implica tener en cuenta la ocurrencia del evento, en qué período se da o la fracción de período y que la ocurrencia de default es irreversible, es decir, no puede salir de la situación independiente de si posteriormente se mejora o recupera del estado default.

Figura 4. Esquema temporal de pagos para el comprador de un CDS

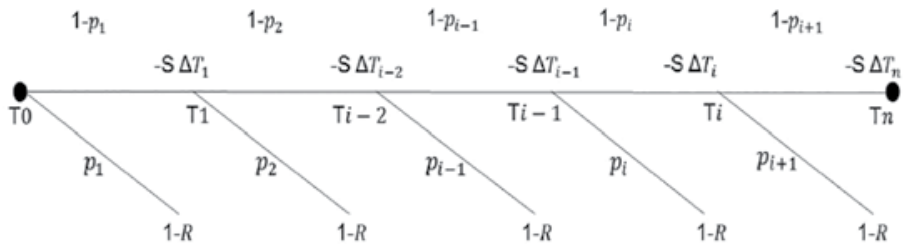

Fuente: Elaboración propia, adaptado de Knop, Hernández, Sánchez y Muñoz (2012).

En este esquema se parte del instante $t_{i-1}$, sobre la base que no hay default, en el siguiente momento $t_{i}$ pueden presentarse dos estados: "vivo" si no ocurrió algún evento de crédito entre el momento $t_{i-1}$ y $t_{i}$; en tanto que la probabilidad de ocurrencia de default será $p_{i}$ entre el momento $t_{i-1}$ y $t_{i}$ asumiendo que llega 
"vivo" hasta $t_{i-1}$ por tanto el modelo de valoración dependerá de los valores de $p_{i}, p_{2} \cdots p_{n}$ que permiten recuperar las probabilidades acumuladas de default de manera recurrente.

La ecuación de valoración con diferentes períodos incluye un término de probabilidad acumulada de default hasta la fecha $T_{i}$, el cual es la probabilidad de que la referencia entre en default antes de la fecha $T_{i}$. De esta manera, $Q_{i}$ se relaciona directamente con las probabilidades condicionales de default, como se aprecia en la siguiente ecuación:

$$
Q_{0}=0 \text { у } Q_{i}=Q_{i-1}+\left(1-Q_{i-1}\right) * p_{i}
$$

Por tanto, la probabilidad de que no entre en default o llegue viva la referencia al período t estará dada por la expresión $\left(1-Q_{i}\right)$. Se puede representar en un árbol la probabilidad para cada uno de los períodos como se muestra en la figura 5.

Figura 5. Esquema temporal de las probabilidades acumuladas de default

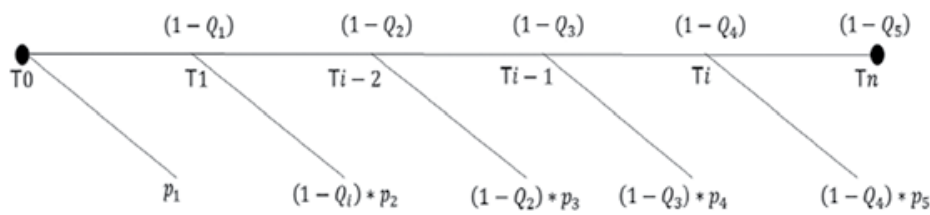

Fuente: Elaboración propia, adaptado de Knop, R., Vidal, J. y Cachán, J. (2003).

Por tanto, a las ecuaciones iniciales 1 y 2 de valoración del CDS se les incorporará la expresión de las probabilidades de incumplimiento acumuladas al vencimiento desde la perspectiva de un comprador, como se presenta de manera formal en las ecuaciones cuatro y cinco a continuación:

\section{Datos de entrada}

El modelo de valoración de Jarrow y Turnbull requiere de las siguientes variables:

- $\quad n$ : Número de pagos de prima convenida.

- $T_{i}$ : Fechas de pago de la prima convenida en el contrato.

- $F \Delta T_{i}$ : Fracción del año entre dos pagos de prima, entre fechas $T_{i-1}$ y $T_{i}$.

- $\quad S$ : Spread (diferencial crediticio) o prima por pagar por parte del comprador de protección en términos anualizados.

- $R$ : Tasa de recuperación estimada.

- $\quad p_{i}$ : Probabilidad de default entre la fecha $i-1$ y la fecha $i$ suponiendo 
el no default anteriormente (denominado probabilidad condicional de incumplimiento entre fechas $T_{i-1}$ y $T_{i}$ ).

- $\quad p_{i p}$ : Probabilidad de default entre la fecha $i-1$ y la fecha $i$ suponiendo el no default anteriormente (aplicable en el caso en que se haya pagado la prima en el momento del default).

- $e^{-r}$ : Factor de descuento sin riesgo al vencimiento del CDS.

- $Q_{i}$ : Probabilidad de default antes de $T_{i}$.

- $\quad\left(1-Q_{i}\right)$ : Probabilidad acumulada de que la referencia no entre en default a $T_{i}$.

Sobre la base del modelo de valoración desde la perspectiva de un comprador, se construye un árbol para cada período de pago de las primas, si se tiene en cuenta que puede suceder el evento de default entre estos períodos, se debe incorporar la fracción de tiempo transcurrida respecto del pago de la prima en la liquidación del CDS y las probabilidades de incumplimiento acumuladas hasta su vencimiento, esto implica ampliar la ecuación inicial del modelo para cada situación posible, lo que se determina en las siguientes expresiones:

Para el caso en el que no se haya pagado la prima, ocurrido el default corresponde:

$$
\begin{aligned}
& C D S=-S * \sum_{i=1}^{n} \Delta T_{i} *\left(1-Q_{i}\right) * e^{-r} \\
& +(1-R) * \sum_{i=1}^{n}\left(1-Q_{i-1}\right) * p_{i} * e^{-r}
\end{aligned}
$$

Para el caso en el que se haya pagado la prima, ocurrido el default corresponde:

$$
\begin{aligned}
& C D S=-S * \sum_{i=1}^{n} \Delta T_{i} *\left(1-Q_{i-1}\right) * e^{-r} \\
& +(1-R) * \sum_{i=1}^{n}\left(1-Q_{i-1}\right) * p_{i} * e^{-r}
\end{aligned}
$$

\section{Cálculos para el análisis}

Para poder aplicar el modelo, es necesario obtener las variables requeridas del modelo y se tienen en cuenta los supuestos, como que la probabilidad condicional de default sea igual para todos los períodos, de allí se puede establecer una expresión que permita obtener la probabilidad de default, relacionando la tasa de recuperación y el spread o prima de mercado, como se presentan las ecuaciones seguidamente.

Como se mencionó anteriormente, bajo el supuesto de que todos los períodos de pago de la prima son iguales $\left(\Delta T_{i}=\Delta T\right)$ y sabiendo que el spread o prima de mercado es el que lleva a cero el valor inicial al CDS (prima en equilibrio $=S_{E}$ ) se llega a la siguiente expresión: 


$$
\begin{aligned}
& 0=-S_{E} * \sum_{i=1}^{n} \Delta T_{i} *\left(1-Q_{i}\right) * e^{-r}+(1-R) * \sum_{i=1}^{n}\left(1-Q_{i-1}\right) \\
& * p_{i} * e^{-r}=-S_{E} * \Delta T * \sum_{i=1}^{n}\left(1-Q_{i}\right) *\left(1-p_{i}\right) * e^{-r}+(1-R) * \\
& \sum_{i=1}^{n}\left(1-Q_{i-1}\right) * p_{i} * e^{-r}=\left[-S_{E} * \Delta T *\left(1-p_{i}\right)+(1-R) * p_{i}\right] \\
& * \sum_{i=1}^{n}\left(1-Q_{i-1}\right) * e^{-r}
\end{aligned}
$$

Para anular este producto, se anula el primer término y se utilizan las siguientes fórmulas para estimar la probabilidad default: Para el caso en el que no haya pagado la prima:

$$
p_{i}=\frac{S_{E} * \Delta T}{1-R+S_{E} * \Delta T}
$$

Para el caso en que se haya pagado la prima en el momento del default:

$$
p_{i p}=\frac{S_{E} * \Delta T}{1-R}
$$

Una de las particularidades en la negociación de CDS es que estos conllevan pagos de primas, por lo general expresados de forma trimestral, y esto difiere de las probabilidades condicionales de default, las cuales por lo regular se expresan sobre periodicidad anual.

Para resolver este inconveniente y poder determinar la probabilidad condicional de default, Knop et al. (2003) manifiestan que se divide el año en períodos iguales, de acuerdo con lo establecido en el CDS y se asume que la probabilidad condicional de default es igual para cada período, debiéndose determinar la probabilidad para los períodos $(1 / \mathrm{n})$ menores al año, esta probabilidad en adelante $p_{n}$.

Por tanto, las probabilidades anuales deben ser equivalentes a las probabilidades periódicas hasta el vencimiento, por lo tanto para un período $t p_{t}$ la probabilidad equivalente será:

$$
p_{t}=1-\left(1-p_{n}\right)^{t}
$$

Donde $p_{a}$ es la probabilidad condicional anual y como resultado la probabilidad para un período $1 / \mathrm{n}$ será:

$$
p_{n}=1-\sqrt[n]{1-p_{a}}
$$

Dadas la limitaciones para la estimación de la prima por la inexistencia de un mercado de derivados de crédito que permita obtener estos datos, se elige por despejar la variable spread o prima o en equilibrio $\left(S_{E}\right)$ de la ecuación (8), donde el valor del spread o prima en equilibrio $\left(S_{E}\right)$ debe resultar cero en el período inicial del CDS, debido a que la ecuación representa una equivalencia, como resultado del despeje se presenta la formula siguiente:

$$
S_{E}=\frac{(1-R) * \sum_{i=1}^{n}\left(1-Q_{i-1}\right) * p^{*} e^{-r}}{\sum_{i=1}^{n} \Delta T_{i} *\left(1-Q_{i}\right) * e^{-r}}
$$




\section{Aplicación empírica - Características de la emisión por cubrir}

En esta sección se presentan los resultados de la aplicación en la valoración de CDS por el modelo Jarrow y Turnbull (1995); en un bono corporativo de una empresa privada colombiana, para lo cual se presenta a continuación una breve reseña del emisor y del activo subyacente a realizar la cobertura de riesgo default.

Con el propósito de valorar el CDS a partir de las variables requeridas por el modelo y después de revisar el mercado de renta fija en Colombia, con las respectivas limitaciones de información de un mercado emergente, se escogió como entidad de referencia a la Empresa Colombiana de Petróleos - Ecopetrol, empresa industrial dedicada a la exploración, explotación, refinación, transporte, almacenamiento, distribución y comercialización de hidrocarburos, sus derivados y productos, por su condición de ser un emisor representativo a nivel nacional, el cual realizó una emisión de bonos de deuda externa en el 2010. Dentro de la emisión, se determinó utilizar la serie $\mathrm{C}$ de bonos que tienen vencimiento a 10 años.

Dentro de esta misma emisión se encuentran clasificados los bonos en unas series que están referenciadas en dólares y otras en moneda local. Las cotizadas en moneda extranjera tienen calificación internacional de calidad de crédito de la emisión $\mathrm{BBB}$, mientras las denominadas en moneda nacional tienen calificación nacional de calidad de crédito AAA; las diferencias entre estas calificaciones distan de manera considerable, y como el estándar del mercado de CDS considera las clasificaciones internacionales, se tomará la calificación internacional asociada al emisor. En la tabla 1 se presenta la información con las principales características del bono de referencia del CDS.

Tabla 1. Datos de la emisión del bono de referencia

\begin{tabular}{ll}
\hline \multicolumn{2}{c}{ Descripción del bono } \\
\hline \multicolumn{1}{c}{ Información del emisor } & \multicolumn{1}{c}{ Valores } \\
\hline Nombre & Ecopetrol \\
Industry & Petroleos integrados \\
\hline Información del valor & \\
\hline Mkt & Global \\
País & CO \\
Ránking & Sr Unsecured \\
Divisa & USD \\
Cupón & 7.625 \\
Tipo & Fljo \\
Frec & Sem \\
Dás & ISMA-30/360 \\
Vence & $07 / 23 / 2019$ \\
Cálculo & $(1)$ Street Convention \\
Fecha de anuncio & $09 / 23 / 2009$ \\
Fecha de devengo & $07 / 23 / 2009$ \\
1ra liquidación & $10 / 02 / 2009$ \\
1er Cupón & $01 / 23 / 2010$ \\
\hline Ratings & \\
\hline Moody's & Baa2 \\
S\&P & BBB \\
Fitch & BBB \\
Composite & BBB \\
\hline Emisión y operación & \\
\hline Emitido/Nigente & $1,496,855.00(M) /$ \\
Minimo incremento & $1,496,855.00(M)$ \\
Nominal & $1,000.00 / 1,000.00$ \\
& $1,000.00$ \\
\hline
\end{tabular}

Fuente: Bloomberg, 2015. 


\section{Aplicación empírica - La valoración del CDS}

En esta primera parte se inicia con la obtención de cada uno de los valores de las fuentes de mercado disponibles, requeridos como datos de entrada del modelo para estimar la prima de mercado, como lo son establecer la tasa de recuperación, la probabilidad default base, la probabilidad default acumulada, el factor de descuento estimación, se tienen presente los respectivos supuestos del modelo para luego con toda la información calcular el valor del CDS.

\section{La tasa de recuperación}

De forma usual, esta variable se estima a partir de datos históricos del comportamiento de pago de los bonos; incluso algunos teóricos asumen valores a priori sin afectar la valoración, así como tampoco influye de manera considerable en las primas del CDS, situación que está demostrada por diversos estudios como el de Hull y White (2000).

Para este trabajo se utilizará como tasa de recuperación el valor de 0,40 utilizada como estándar a nivel internacional para el emisor, como se comprueba en la figura 6 con la información general del contrato para el bono elegido, obtenido en la plataforma Bloomberg. En ella se resaltan con un óvalo los ítems "tipo rec" y "tasa recup", ambos correspondientes al valor de la tasa de recuperación y equivalente al $40 \%$, para la fecha del 21 de enero de 2015. Este valor se asumirá constante en el tiempo en este estudio.

Figura 6. Datos generales del bono para la negociación del CDS

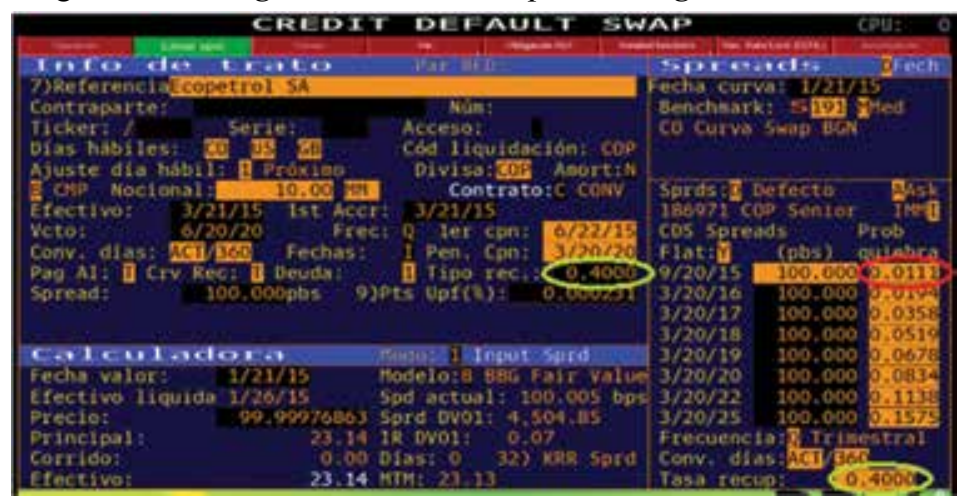

Fuente: Bloomberg (2015).

\section{La probabilidiad de default}

El modelo propone despejar la probabilidad a partir de la formula (8), sustituyendo el spread de equilibrio por el utilizado en el estándar del mercado de CDS (100 
puntos básicos), como se evidencia en la figura 6 obtenido de la plataforma Bloomberg en la fecha de estudio. Para determinar qué tanto la probabilidad así obtenida se ajusta a la realidad, se la compara con el valor actual del mercado que proyecta como primera fecha de estimación para la probabilidad el 20 de septiembre de 2015 cuya fracción de año será equivalente a (8/12). Reportado por Bloomberg en la fecha del estudio. En efecto, la probabilidad estimada es 0.0111 , que coincide exactamente con el valor reportado por Bloomberg como se aprecia resaltado en un círculo rojo en la figura 6.

Por consiguiente, la estimación de la probabilidad condicional de default periódica se obtiene a partir del uso de la probabilidad de default anual (para este caso proporcional según las fechas determinadas por el mercado), en este caso 0.011. Esta probabilidad, llevada a trimestres a partir de (10), arroja como resultado el valor de 0.00278663 . En consecuencia se hallan las demás probabilidades acumuladas de default de los siguientes períodos con la aplicación de la ecuación número 3 , como se relacionan en la tabla 2 .

Tabla 2. Probabilidades acumuladas para periodos trimestrales

\begin{tabular}{cccccc}
\hline Período & Probabilidad & Período & Probabilidad & Período & Probabilidad \\
\hline Q0 & 0.0000 & Q7 & 0.019344 & Q14 & 0.038314 \\
Q1 & 0.002787 & Q8 & 0.022077 & Q15 & 0.040994 \\
Q2 & 0.005565 & Q9 & 0.024802 & Q16 & 0.043666 \\
\hline Q3 & 0.008337 & Q10 & 0.027519 & Q17 & 0.046331 \\
\hline Q4 & 0.011100 & Q11 & 0.030229 & Q18 & 0.048989 \\
Q5 & 0.013856 & Q12 & 0.032932 & Q19 & 0.051639 \\
\hline Q6 & 0.016604 & Q13 & 0.035627 & Q20 & 0.054282 \\
\hline
\end{tabular}

Fuente: Elaboración propia.

\section{El factor de descuento}

Para el factor de descuento se utilizó la información al 21 de enero de 2015 de la curva de la tasa libre de riesgo en Colombia, específicamente la tasa cero cupón con vencimiento a cinco años. Esta decisión se justifica en razón a que esta es equivalente al tiempo restante del vencimiento del bono de referencia que se desea cubrir. El resultado obtenido es $5.487 \%$ tasa calculada y publicada por el Banco de la República de Colombia para la misma fecha como se observa en la figura 7. 
Figura 7. Curva de rendimientos soberanos cupón cero

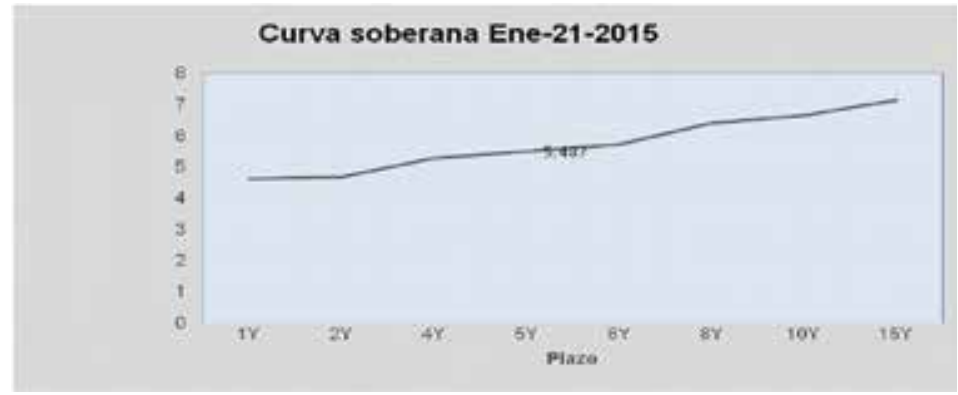

Fuente: Elaboración propia con datos del Banco de la Republica de Colombia.

\section{El spread}

Debido a la inexistencia de un mercado de derivados de crédito que permita deducir la prima, se planteó obtenerlo a partir de despejar la prima en equilibrio variable (SE) de la ecuación 8. El resultado es cero en el período inicial o tiempo cero del CDS, debido a que los dos componentes de la ecuación deben ser equivalentes para llegar a la ecuación 11.

Al remplazar todos los datos en la ecuación 11, se hacen los cálculos y se obtiene el valor de la prima en equilibrio de 0,668790313. Por tanto, al momento de cotizar en el mercado el valor del CDS para el bono de Ecopetrol, el dato obtenido a partir de las variables utilizadas en el modelo será el valor de referencia de la prima que se negocie, es decir, aproximadamente 67 puntos básicos, y en ella cualquier diferencia representará un mayor o menor valor del contrato y, por tanto, un beneficio para el comprador o el vendedor según sea el caso.

\section{Análisis de la valoración}

Para llevar a cabo la valoración del CDS se requiere establecer los valores de los restantes datos de entrada del modelo, a continuación, se presenta cada una:

El número de pagos de prima convenida de acuerdo con el estándar del mercado serán de cuatro por cada año para un total de 20 pagos en cinco años, lo que corresponde a períodos de pagos trimestrales, es decir, $n=1 / 4=0.25$ como usualmente predomina en la estructura de pagos de los contratos de CDS negociados a nivel mundial.

Las fechas de pago de las primas serán referenciadas a partir de las fechas de pago de los cupones del bono de referencia. 
El valor nominal que se utiliza de referencia será de diez millones $(\$ 10.000 .000)$ y el valor de la prima anual será del uno por ciento (1\%) o cien puntos básicos del valor del nominal según los estándares del mercado pagaderos de forma trimestral, convirtiéndose en $\$ 25.000$.

Al desarrollar el modelo de valoración sobre la base de un spread de $1 \%$ o cien puntos básicos, se obtiene como resultado, desde el punto de vista del comprador, un valor de \$140.272,54 lo que significa, como ya se había inferido, que el precio no está en equilibrio. El valor resultante favorece a la posición vendedora del CDS; esto se relaciona con el resultado del valor presente esperado de los flujos de las primas, mayor al valor presente de los flujos esperados para recuperar por parte del emisor. En caso de no obtener un valor positivo, el valor del CDS favorecería a la posición compradora de la protección del CDS. La manera como se obtuvo este resultado, fue con el desarrollo de un modelo financiero utilizando la herramienta de Microsoft Excel, el cual estructuró para simultáneamente obtuviera los valores presentes de cada rama según se presente default y para cuando no se presente default, previamente realizando operaciones como la multiplicación de la fracción del pago de la prima por la probabilidad acumulada que a su vez multiplica el factor de descuento y le suma la diferencia de la tasa de recuperación que multiplica las probabilidades acumuladas por la probabilidad de default y al factor de descuento, para al final establecer el valor que arroja el modelo como la diferencia entre las dos ramas.

Al comparar el resultado obtenido en el modelo financiero al recalcular el spread o prima requerida que permite un valor en equilibrio se obtiene un resultado de 66,8790 puntos básicos comparado frente a la valoración del mercado, se encuentra que la plataforma Bloomberg un valor de 60,6672 puntos básicos de spread del CDS de referencia a corte del mercado del 20 de septiembre de 2015, es muy cercano al valor obtenido. La diferencia existente, esta sobre los seis puntos básicos y esto se puede explicar en razón a protocolos del mercado que no contempla el modelo teórico, tales como períodos estándar para liquidar los spreads y las probabilidades que se ajustan según las fechas de pago, afectadas por el estándar de las fechas de coberturas que inician 60 días antes de la fecha de adquisición del CDS.

\section{Conclusiones}

A partir de los resultados obtenidos se aprecia que el cálculo del spread por el modelo utilizado presentó un valor superior cercano a los quince puntos básicos; al ajustar del cálculo de la proporción de la probabilidad de default anual de acuerdo con los protocolos de negociación del mercado se encontró una diferencia aproximada de 6,21 puntos básicos. Esta información sería útil para que el inversionista del mercado de bonos con fines de cobertura contraste el valor teórico obtenido del modelo contra el spread al cual se encuentre negociando el CDS de referencia y tome las decisiones de negociación. 
Se evidencia que el modelo de Jarrow y Turnbull presenta un alto grado de aproximación con los datos utilizados a cinco años, al comparar los datos del modelo contra los datos del mercado, encontrándose ajustado para obtener la probabilidad anual base, aunque tiene un desfase mínimo con respecto de la estimación de las probabilidades acumuladas, por tanto, se considera viable y adecuado la aplicación del modelo para la estimación de la probabilidad default acumulada de CDS, lo que serviría a aquellos futuros inversionistas que deseen cubrir los riesgos y que no disponen de herramientas, ni de suficientes datos del mercado para medir la probabilidad default.

Se observó que las variables que ocasionan un desfase mínimo en el cálculo en el modelo de valoración aplicado, se debe a que el modelo no incorpora variables que se encontraban en la pantalla de negociación, las cuales son producto de los estándares o protocolos de negociación internacional, entre los que se encontraron: las fechas de pago del spread y el concepto del pago Upfront, los cuales afectan directamente el cálculo de la probabilidad default, variables estas que no se contempla en el modelo de valoración.

Se puede aplicar modelo de Jarrow y Turnbull para estimar probabilidades default como las primas o spread del CDS lo que permita valorar y gestionar activamente el riesgo default y el riesgo de spread en emisiones de deuda privada en Colombia que cuenten con la información requerida en el modelo, y considerar el efecto de descalce que se pueda presentar por las diferencias que puedan existir entre la frecuencia de pago de los cupones y la frecuencia trimestral del pago de la prima del CDS.

Este estudio arroja luz sobre un tema poco cubierto en Colombia, el cual es la valoración y cobertura de CDS en bonos corporativos. Como se indicó, los resultados demuestran la conveniencia de este tipo de derivados en la gestión de coberturas de riesgo de default y en Colombia se necesita un cambio en la normativa que permita su aplicación y el estudio y difusión de resultados que, como este, demuestran la viabilidad de propuestas de este tipo.

Los CDS permitirían promover el crecimiento y la inversión del mercado de bonos corporativos al permitir la obtención de un rendimiento estable sin cambiar la calificación de riesgo; incluso podrían, en algunos casos, ser más ventajosos en la relación riesgo rendimiento frente a bonos de Gobierno, considerados libres de riesgo de crédito, en condiciones de vencimiento y pagos similares.

El modelo teórico de Jarrow y Turnbull requiere realizar una adaptación para adecuarlo a los protocolos del mercado en lo correspondiente a las fechas para estimar la probabilidad default de base y las probabilidades acumuladas, con el fin de que los resultados se ajusten con mayor precisión a los valores del mercado.

El desarrollo de este trabajo plantea interrogantes adicionales respecto del planteamiento del comportamiento y uso de las variables para el análisis, tanto de la probabilidad default como de la valoración de derivados de crédito que podrían ser objeto de otras investigaciones que complementen los resultados hasta aquí obtenidos. 


\section{Referencias}

Arbeláez, J. C., \& Maya, C. (2007). Valoración de Credit Default Swaps (CDS): Una Aproximación Montecarlo. Cuadernos de Administración, 1, 87.

Arce, O., González, J., y Sanjuán, L. (2010, octubre). El mercado de Credit Default Swaps: Áreas de vulnerabilidad y respuestas regulatorias. CNMV Comision Nacional del Mercado de Valores. Documento de trabajo número 42. Recuperado el 17 de agosto de 2014, de Comision Nacional del Mercado de Valores de España:

http://www.cnmv.es/DocPortal/Publicaciones/MONOGRAFIAS/MonografiaN42 web.pdf

Bonás A., Llanes, M., Usón, I., y Veiga, N. (2007). Riesgo de crédito amenaza u oportunidad, (pp. 29-32). Universitat Pompeu Fabra - IDEC.

Conde, G., Malacrida, F., y Selves, R. (2003). Valuación de instrumentos sujetos a riesgo de crédito. Biblioteca Virtual del Banco Central del Uruguay, 8, 1-38.

http://www.bvrie.gub.uy/local/File/JAE/2003/iees03j3370803.pdf

Duffie, D., y Singleton, K. J. (1999). Modeling Term Structures of Defaultable Bonds. The Review of Financial Studies, vol. 12, 687-720. http://web.stanford. edu/ duffie/jstorlinks/ds2.pdf

Fradique-Méndez, C. (2008). Aspectos legales de los contratos marco para derivados. Revista de Derecho Privado, vol. 39, 6-7.

Hull, J. C., y White, A. (2000). Valuing Credit Default Swaps I: No Counterparty Default Risk. Journal of Derivatives, vol. 8, 29-40.

International Swaps and Derivatives Association. (2015, agosto 1). International Swap and Derivatives Association. Recuperado de ISDA: http://www2.isda.org/

International Swaps and Derivatives Association. (s.f.). ISDA. Recuperado el 29 de septiembre de 2014, de http://www2.isda.org

Jarrow, R., y Turnbull, S. (1995). Pricing derivatives on financial securities subject to credit risk. The Journal of Finance, 50(1), 53-85.

Jarrow, R., Lando, D., y Turnbull, S. (1997). A markov model for the term structure of credit risk spreads. Review of Financial Studies, vol. 10, 481-523.

Knop, R., Hérnandez, R., Sánchez, D., y Muñoz, L. (2012). Credit Defualt Swap. Madrid: Delta Publicaciones.

Knop, R., Vidal, J., y Cachán, J. (2003). Derivados de crédito. Aspectos financieros y legales. Madrid: Bibilioteca Fundación de Estudios Financieros - Ediciones Pirámide. 
Labanda, A. (2005). Derivados de riesgo de crédito, tipos y utilidades. Documento de trabajo 0506 Universidad Complutense. Recuperado de https://www.ucm.es/ data/cont/media/www/pag-19529/Publi.\%20Mascare\%C3\%Blas/0506.pdf

Pérez, J. (2002). Los derivados de crédito. Estabilidad Financiera Banco de España, vol. 3, 59-83. Recuperado de http://www.bde.es/f/webbde/Secciones/ Publicaciones/InformesBoletinesRevistas/RevistaEstabilidadFinanciera/02/Fic/ estfin03.pdf

Sasaki, H. H., Chela, J. L., \& Kimura, H. (2012). Metodología para Precificação de Credit Default Swaps. Revista de Economía Mackenzie, vol 7, 4-23. 